\title{
Efeito de herbicidas aplicados em jato dirigido no desenvolvimento inicial de plantas de pinhão-manso ${ }^{1}$
}

\section{Effect of herbicides applied as direct spray in the initial development of jatropha plants}

Neumarcio Vilanova Costa $^{2}$; Tania Helena Neunfeld ${ }^{3}$; Tatiane Ohland ${ }^{4}$; Jeferson Tiago Piano ${ }^{4}$; Andreia Cristina Peres Rodrigues-Costa ${ }^{5}$

Resumo - Objetivou-se avaliar o efeito de glyphosate, paraquat e do amônio-glufosinato aplicados em plantas de pinhão-manso. Os tratamentos consistiram: glyphosate $(180,360,720 \mathrm{e}$ $\left.1.440 \mathrm{~g} \mathrm{ha}^{-1}\right)$, paraquat $\left(100,200,400\right.$ e $\left.800 \mathrm{~g} \mathrm{ha}^{-1}\right)$ e amônio-glufosinato $(125,250,500 \mathrm{e} 1.000 \mathrm{~g}$ $\mathrm{ha}^{-1}$ ) e testemunha (sem aplicação). A aplicação dos herbicidas foi realizada aos 145 dias após a semeadura, em jato dirigido no terço médio inferior das plantas. As doses de glyphosate proporcionaram médias de fitointoxicação de $2,4 \%$ e não houve redução da área foliar, contudo, constatou-se redução média da massa seca de $9,0 \%$ aos 35 dias após a aplicação (DAA). Para o paraquat, até a dose de $200 \mathrm{~g} \mathrm{ha}^{-1}$ ocorreram fitointoxicações inferiores a $20 \%$ até os 35 DAA, entretanto, houve redução da área foliar e da massa seca das plantas de 47,0 e 18,2\%, respectivamente. A dose de $125 \mathrm{~g} \mathrm{ha}^{-1}$ de amônio-glufosinato proporcionou menores valores de fitointoxicação $(<20,0 \%)$, de redução da área foliar $(2,7 \%)$ e da massa seca $(10,5 \%)$ até 35 DAA. Concluiu-se que o contato do paraquat e do amônio-glufosinato com o caule e folhas pode prejudicar o desenvolvimento inicial das plantas. Entretanto, a aplicação do glyphosate não prejudicou o desenvolvimento inicial das plantas de pinhão-manso.

Palavras-chaves: Jatropha curcas, seletividade a herbicidas, fitointoxicação

Abstract - The objective was to evaluate the effect of glyphosate, paraquat and glufosinateammonium applied in jatropha plants. The treatments consisted: glyphosate $(180,360,720$ and 1,440 $\left.\mathrm{g} \mathrm{ha}^{-1}\right)$, paraquat $\left(100,200,400\right.$ and $\left.800 \mathrm{~g} \mathrm{ha}^{-1}\right)$ and glufosinate-ammonium $(125,250,500$ and $1,000 \mathrm{~g} \mathrm{ha}^{-1}$ ) and a control (no application). Herbicide application was performed at 145 days after sowing, with spraying directed to the lower third middle of the plants. The rates glyphosate provided average phytointoxication of $2.4 \%$ and no reduction in leaf area, however, there was an average reduction of $9.0 \%$ of dry weight at 35 days after application (DAA). Paraquat, for rates $\leq$ $200 \mathrm{~g} \mathrm{ha}^{-1}$, phytointoxication was lower than $20 \%$ to 35 DAA, however, there was a reduction in

\footnotetext{
${ }^{1}$ Recebido para publicação em 15/01/2014 e aceito em 10/05/2015.

${ }^{2}$ Docente do curso de Agronomia da Universidade Estadual do Oeste do Paraná (UNIOESTE). R. Pernambuco, 1.777 - Centro - Caixa Postal 91, 85960-000, Marechal Cândido Rondon, Paraná, Brasil. Email: neumarciovc@ hotmail.com (*autor para correspondência).

3 Doutoranda do Programa de Pós-graduação em Agronomia da Universidade Estadual do Centro-Oeste (UNICENTRO), Campus CEDETEG, Guarapuava, Paraná.

${ }^{4}$ Doutorandos do Programa de Pós-Graduação em Agronomia da Universidade Estadual do Oeste do Paraná (UNIOESTE), Campus Marechal Cândido Rondon, Marechal Cândido Rondon, Paraná.

${ }^{5}$ Docente do curso de Agronomia da Universidade Estadual de Maringá (UEM), Campus Regional de Umuarama, Estrada da Paca, Bairro São Cristóvão, 87507190, Umuarama, Paraná.
} 
leaf area and plant dry mass of 47.0 and $18.2 \%$, respectively. The rate of $125 \mathrm{~g} \mathrm{ha}^{-1}$ glufosinateammonium was the one that gave the lowest values of phytointoxication $(<20.0 \%)$, reduction in leaf area $(2.7 \%)$ and dry mass $(10.5 \%)$ plants up to 35 DAA. It was concluded that the contact of paraquat and glufosinate-ammonium with stems and lower leaves may impair the initial development of jatropha. However, application of glyphosate direct spray did not impair the initial development of jatropha.

Keywords: Jatropha curcas, selectivity of herbicide, phytotoxicity

\section{Introdução}

O pinhão-manso é uma oleaginosa perene que se destaca como alternativa importante para a produção de biodiesel. Entretanto, para a exploração industrial do óleo torna-se necessário obter informações sobre seu cultivo de modo que todo o potencial da cultura possa ser maximizado (Openshaw, 2000; Achten et al., 2008; Kumar \& Sharma, 2008; Divakara et al., 2010).

Desta forma, semelhante a outras culturas perenes, as plantas jovens do pinhãomanso podem sofrer interferência negativa da comunidade das plantas daninhas e ter o seu crescimento e desenvolvimento vegetativo comprometido, bem como a produção de sementes e de óleo (Erasmo et al., 2009; Fey et al., 2013; Duarte Júnior et al., 2013; Silva et al., 2013). Assim, durante a fase inicial de desenvolvimento das plantas de pinhão-manso é necessário a adoção de medidas de manejo das plantas daninhas (Saturnino et al., 2005; Rocha et al., 2010).

Dentre os métodos de manejo das plantas daninhas utilizados em culturas perenes, o uso de herbicidas não seletivos aplicados em jato dirigido nas plantas daninhas pode ser uma excelente alternativa, considerando principalmente o manejo dessas espécies em extensas áreas de plantio (Costa et al., 2013). Porém, existem poucos estudos sobre o uso de herbicidas com distintos mecanismos de ação para o controle das plantas daninhas na cultura do pinhão-manso, por meio de tecnologias de aplicação em jato dirigido, as quais permitam viabilizar a seletividade de produtos de ação total disponíveis no mercado.
De acordo com Costa et al. (2009), plantas de pinhão-manso apresentaram tolerância à deriva simulada de glyphosate, o que evidencia o potencial de utilização deste herbicida no manejo das plantas daninhas em aplicações com jato dirigido. Em outro estudo, Costa et al. (2013) constataram que o paraquat aplicado em jato dirigido apresentou bom potencial de seletividade para plantas de pinhãomanso.

Considerando estes resultados, o amônio-glufosinato que também é um herbicida de ação total e disponível no mercado brasileiro poderia ser utilizado em jato dirigido no controle das plantas daninhas na cultura do pinhão-manso.

Destaca-se ainda que a utilização de herbicidas com mecanismos de ação distintos pode auxiliar no controle das plantas daninhas em áreas que apresentam alta variabilidade de espécies, uma vez que, nessa condição, a utilização de apenas um tipo de herbicida pode promover controle ineficiente e aumentar a pressão de seleção sobre espécies resistentes aos herbicidas (Christoffoleti \& Lopez-Ovejero, 2003; Beckie \& Reboud, 2009; Vencill et al., 2012).

Portanto, as hipóteses do presente trabalho baseiam-se no fato de que a utilização de herbicidas que apresentam mecanismos de ação distintos, como o glyphosate (inibidor da EPSPs), o paraquat (inibidor do fotossistema I) e do amônio-glufosinato (inibidor da glutamina sintetase) pode reduzir a pressão de seleção de biótipos resistentes e conferir maior eficiência no manejo das plantas daninhas. Assim como, a seletividade para a cultura do pinhão-manso dos 
herbicidas de ação total poderia ser conferida pela aplicação com jato dirigido.

Dessa maneira, objetivou-se avaliar o efeito do glyphosate, do paraquat e do amônioglufosinato aplicados em jato dirigido no desenvolvimento inicial de plantas de pinhãomanso.

\section{Material e Métodos}

O estudo foi conduzido em viveiro telado (sombrite $50 \%$ de luminosidade) com delineamento experimental em blocos casualizados, com cinco repetições. Os tratamentos consistiram-se na avaliação dos seguintes herbicidas e doses: glyphosate (180, 360,720 e $\left.1.440 \mathrm{~g} \mathrm{ha}^{-1}\right)$, paraquat $(100,200,400$ e $\left.800 \mathrm{~g} \mathrm{ha}^{-1}\right)$ e amônio-glufosinato $(125,250$, 500 e $1.000 \mathrm{~g} \mathrm{ha}^{-1}$ ) e uma testemunha (sem aplicação).

Foram utilizados vasos plásticos com capacidade de $17,81 \mathrm{dm}^{3}$. O solo apresentava as seguintes características físicas: $143 \mathrm{~g} \mathrm{~kg}^{-1} \mathrm{de}$ argila, 35,95 $\mathrm{g} \mathrm{kg}^{-1}$ de silte e $821,05 \mathrm{~g} \mathrm{~kg}^{-1}$ de areia, tendo como resultado da análise química os valores de $\mathrm{pH}$ em $\mathrm{CaCl}_{2}$ de 4,85; 6,15 $\mathrm{g} \mathrm{dm}^{-3}$ de MO; 29,93 $\mathrm{g} \mathrm{dm}^{-3}$ de P; 0,10 $\mathrm{cmol}_{\mathrm{c}} \mathrm{dm}^{-3}$ de $\mathrm{K} ; 0,95 \mathrm{cmol}_{\mathrm{c}} \mathrm{dm}^{-3}$ de Ca $0,29 \mathrm{cmol}_{\mathrm{c}} \mathrm{dm}^{-3}$ de $\mathrm{Mg}$, 0,20 $\mathrm{cmol}_{\mathrm{c}} \mathrm{dm}^{-3}$ de Al+3 4,48 $\mathrm{cmol}_{\mathrm{c}} \mathrm{dm}^{-3}$ de CTC e 29,91 de V\%. O solo foi corrigido para $60 \%$ de saturação de bases.

A semeadura foi realizada em 18 de outubro de 2010, com cinco sementes por vaso, sendo que aos quinze dias após a semeadura (DAS) foi realizado o desbaste, permanecendo apenas uma planta por vaso. A adubação nitrogenada de cobertura foi realizada aos $15 \mathrm{e}$ 30 DAS, utilizando-se 11,6 gramas de uréia por vaso.

A aplicação dos herbicidas foi realizada aos 145 DAS com auxílio de um pulverizador costal, pressurizado a $\mathrm{CO}_{2}$ e equipado com ponta Jacto F110/08/3 (110/LD/02), com pressão de $22 \mathrm{Lb}$ pol$^{-2}$ e regulado para proporcionar volume de calda de $200 \mathrm{~L} \mathrm{ha}^{-1}$ a velocidade de $3,6 \mathrm{~km} \mathrm{~h}^{-1}$. As condições ambientais na aplicação foram: Temperatura de $25^{\circ} \mathrm{C}$, umidade relativa do ar de $70 \%$ e velocidade do vento de $6 \mathrm{~km} \mathrm{~h}^{-1}$. Os vasos foram dispostos linearmente em um metro e a aplicação foi realizada com jato fixo direcionado as folhas e caule do terço médio inferior de ambos os lados das plantas.

Aos 7, 14, 21, 28 e 35 dias após a aplicação (DAA) realizou-se a avaliação da fitointoxicação das plantas causadas pelos herbicidas. Foram atribuídas notas visuais, de acordo com escala percentual, na qual 0 correspondeu à ausência de intoxicação e 100 à morte das plantas (SBCPD, 1995). Para a atribuição das notas considerou-se a inibição do crescimento, quantidade e uniformidade das injúrias, abscisão foliar e capacidade de rebrota das plantas.

Aos 35 DAA avaliou-se a área foliar $\left(\mathrm{cm}^{2}\right)$ utilizando-se a fórmula $\mathrm{AF}=0,84[\mathrm{CxL}]^{0,99}$, proposta por Severino et al. (2007), em que: $C=$ comprimento da região central do limbo foliar e $\mathrm{L}=$ largura da região mais extensa do limbo foliar. $\mathrm{Na}$ mesma ocasião, foi determinada a massa seca (g) das plantas, sendo o material vegetal coletado fracionado e acondicionado em sacos de papel e levado à estufa de circulação de ar a $65^{\circ} \mathrm{C}$ por 72 h.

Os dados obtidos de fitointoxição foram ajustados a modelos múltiplos de regressão e a gráficos de superfície de resposta. Enquanto que as médias da área foliar e de massa seca das plantas foram ajustadas a modelos de regressão, sendo as equações escolhidas com base nos modelos significativos, com lógica biológica e de elevado $\mathrm{R}^{2}$.

\section{Resultados e Discussão}

$\mathrm{Na}$ Figura 1, estão apresentados os resultados de porcentagem de fitointoxicação nas plantas de pinhão-manso após a aplicação dos herbicidas. Pode-se observar que as doses de glyphosate causaram fitointoxicação leve $(2,4 \%)$ nas plantas de pinhão-manso até os 35 DAA. O uso de paraquat em doses até $200 \mathrm{~g} \mathrm{ha}^{-}$ ${ }^{1}$ causou fitointoxicação de $20 \%$, sendo que 
quando se utilizaram as doses de 400 e $800 \mathrm{~g} \mathrm{ha}^{-}$ ${ }^{1}$ a fitointoxicação ocorreu na ordem de 40 e $60 \%$, respectivamente, aos 35 DAA. O amônioglufosinato nas doses inferiores a $500 \mathrm{~g} \mathrm{ha}^{-1}$ promoveu fitointoxicação de até $20 \%$ nas plantas de pinhão-manso, enquanto que na dose de $1.000 \mathrm{~g} \mathrm{ha}^{-1}$ os sintomas de fitointoxicação foram de até $40 \%$.

Os sintomas de fitointoxicação causados pela aplicação do glyphosate corresponderam ao amarelecimento de algumas folhas da região aplicada da planta. Os sintomas causados pelo paraquat caracterizaram-se pela formação de pontos cloróticos nas folhas com posterior escurecimento da área, devido à necrose do tecido. Houve também a formação de pontos necrosados na parte do caule que recebeu aplicação. Ainda neste tratamento, houve abscisão foliar na região aplicada da planta, ocorrendo abscisão total das folhas da planta em doses superiores a $800 \mathrm{~g} \mathrm{ha}^{-1}$ aos 21 DAA.

$\mathrm{O}$ amônio-glufosinato causou a formação de áreas cloróticas seguidas de necrose do tecido foliar, típicos de produtos de ação de contato. De forma, semelhante ao paraquat, houve abscisão das folhas até a região mediana da planta.

A abscisão foliar causada pelo paraquat e amônio-glufosinato pode ser justificada devido às folhas terem recebidos os maiores depósitos de calda, diretamente ou por deriva, no momento da aplicação, o que pode ter favorecido a absorção do produto. Portanto, estes resultados indicam que para a utilização do paraquat e do amônio-glufosinato em jato dirigido na cultura do pinhão, é necessário o uso de equipamentos de proteção (Chapéu-denapoleão) que impeçam o contato do jato com o caule e as folhas do terço médio inferior das plantas.

Para a cultura da mamoneira de porte anão, a aplicação de paraquat + diuron $\left(\right.$ Gramocil $^{\circledR}-3,0 \mathrm{~L} \mathrm{ha}^{-1}$ ) sem o uso do chapéude-napoleão proporcionou maior fitointoxicação nas plantas, sendo observado aos 10, 20 e 30 DAA que as plantas apresentavam, respectivamente, 51, 76 e 72\% de fitointoxicação para o tratamento sem o chapéude-napoleão e 26, 43 e $40 \%$ para o tratamento com o uso deste equipamento de proteção (Maciel et al., 2008).

Segundo Costa et al. (2013), misturas do paraquat (200 e $\left.600 \mathrm{~g} \mathrm{ha}^{-1}\right)$ com diuron $(1.000 \mathrm{e}$ $2.000 \mathrm{~g} \mathrm{ha}^{-1}$ ) conferiram prejuízo ao desenvolvimento e à atividade fotossintética das plantas de pinhão-manso em aplicações com jato dirigido, enquanto que o paraquat aplicado isolado nas dosagens de 200 e $600 \mathrm{~g} \mathrm{ha}^{-1}$ em jato dirigido apresentou bom potencial de seletividade para plantas de pinhão-manso. Ressalta-se que estes resultados divergem dos obtidos no presente trabalho.

De acordo com Costa et al. (2009), plantas de pinhão-manso apresentaram boa tolerância à deriva simulada de glyphosate até a dose de 22,5 $\mathrm{g} \mathrm{ha}^{-1}$. Provavelmente as plantas de pinhão-manso apresentam a capacidade de metabolizar o glyphosate em baixas concentrações ou de imobilizá-lo no vacúolo. Assim, a aplicação em jato dirigido pode reduzir o contato do glyphosate com a planta e, consequentemente, conferir maior segurança nas aplicações deste herbicida às plantas de pinhão-manso.

Gonçalves et al. (2011) aplicando glyphosate nas dosagens de 180 a $1.260 \mathrm{~g} \mathrm{ha}^{-1}$ em jato dirigido em plantas de pinhão-manso, verificaram 55 dias após a semeadura, que apenas a dosagem de $180 \mathrm{~g} \mathrm{ha}^{-1}$ de glyphosate apresentou-se seletiva ao pinhão-manso, mostrando-se promissora para o controle de plantas daninhas em lavouras jovens.

Quanto à área foliar, não foram observados efeitos negativos após a aplicação do glyphosate (Figura 2 - A), enquanto que, nas aplicações de paraquat verificou-se redução da área foliar em 45,2; 47,0; 53,5 e 71,4\% para as doses de 100, 200, 400 e $800 \mathrm{~g} \mathrm{ha}^{-1}$, respectivamente (Figura 2 - B).

A aplicação de amônio-glufosinato nas doses de 125, 250, 500 e $1000 \mathrm{~g} \mathrm{ha}^{-1}$ reduziu a área foliar na ordem de 10,5; 36,6; 45,0 e 64,4\%, 
respectivamente (Figura 2 - C). Estes resultados corroboram com a ocorrência da abscisão foliar verificada após as aplicações do paraquat e do amônio-glufosinato.

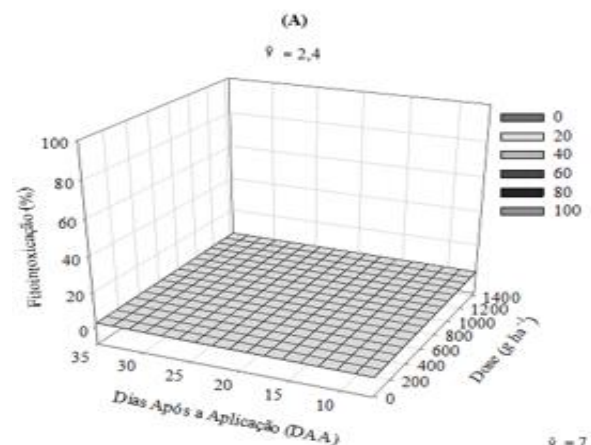

(C)
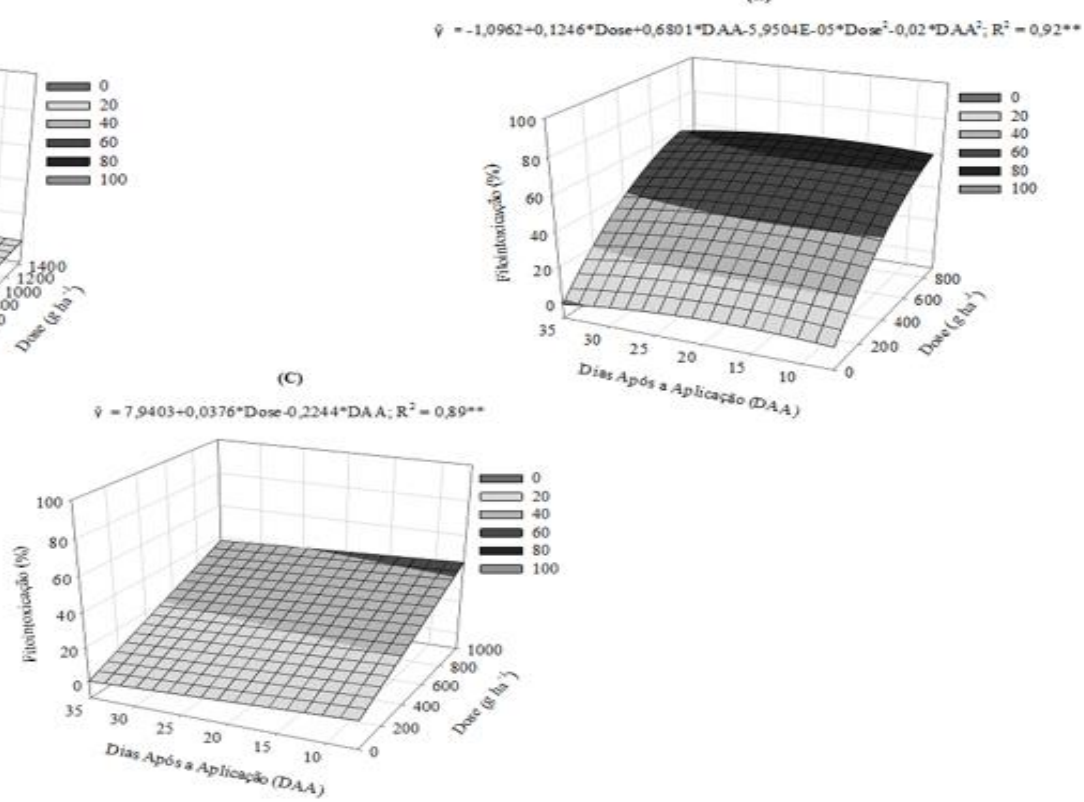

**significativo a $1 \%$ de probabilidade pelo teste $\mathrm{F}$.

Figura 1. Porcentagem de fitointoxicação nas plantas de pinhão-manso após a aplicação dos herbicidas. (A) glyphosate, (B) paraquat e (C) amônio-glufosinato.
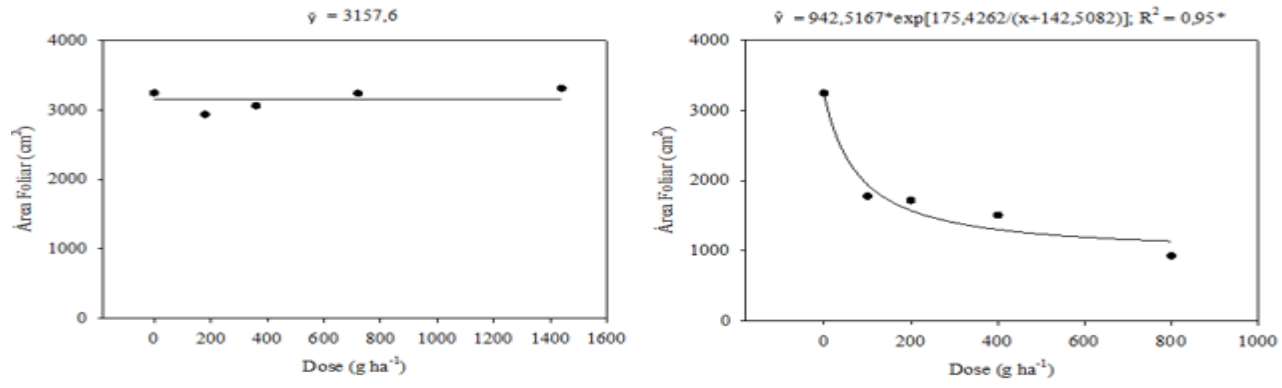

(C)

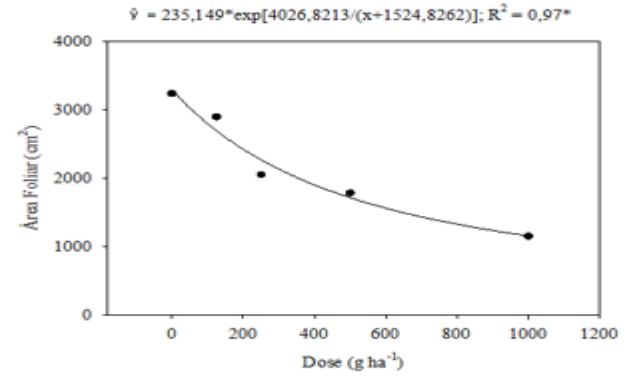

**significativo a $1 \%$ de probabilidade pelo teste $\mathrm{F}$.

Figura 2. Área foliar das plantas de pinhão-manso após a aplicação dos herbicidas aos 35 DAA. (A) glyphosate, (B) paraquat e (C) amônio-glufosinato.

Comportamento similar aos de área produção de massa seca das plantas, ressaltando foliar também foi verificado para os dados de que o glyphosate na maior dose proporcionou 
menor redução $(9,0 \%)$ em relação às maiores doses do paraquat $(37,3 \%)$ e do amônioglufosinato $(29,9 \%)$ (Figura 3).

Dessa forma, destaca-se a possibilidade de utilização de glyphosate em aplicações de jato dirigido na cultura do pinhão-manso para o manejo das plantas daninhas sem o uso de equipamento de proteção como o chapéu-de- napoleão, ao contrário do verificado para a utilização do paraquat e do amônio-glufosinato.

Contudo, ressalta-se a importância da realização de estudos que visem aprimorar a tecnologia de aplicação do paraquat e do amônio-glufosinato, de modo a se evitar os danos causados no desenvolvimento das plantas.
(A)

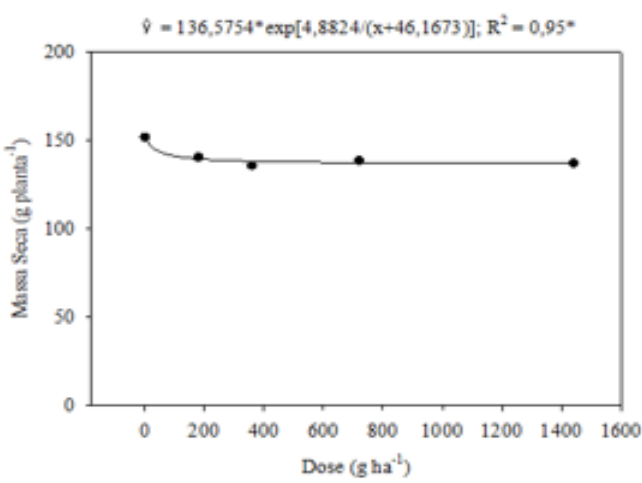

(B)

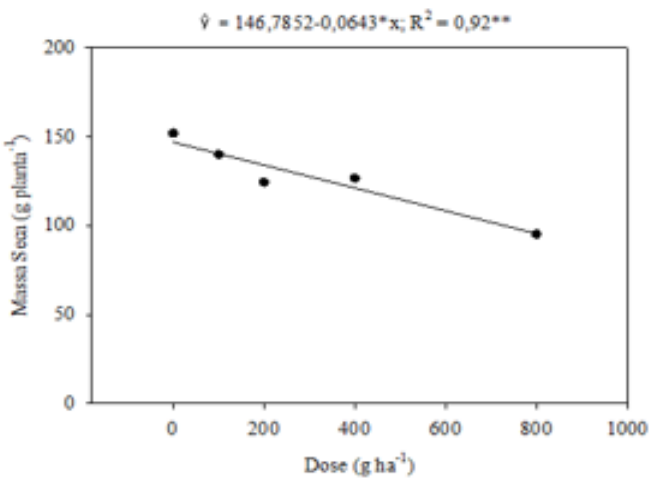

(1)

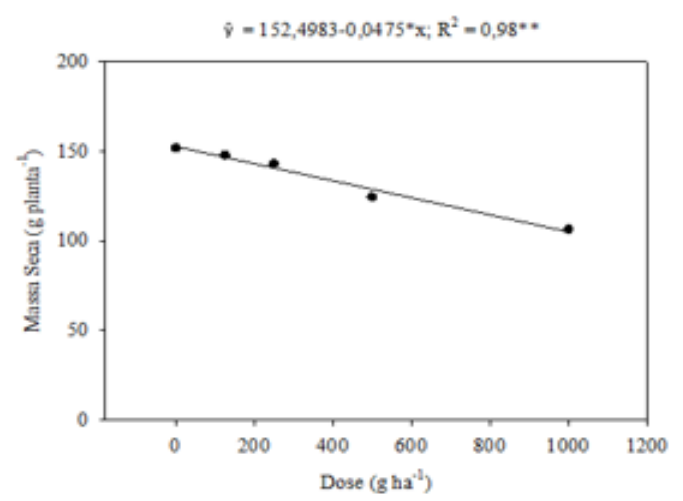

**significativo a $1 \%$ de probabilidade pelo teste $\mathrm{F}$.

Figura 3. Massa seca das plantas de pinhão-manso após a aplicação dos herbicidas aos 35 DAA. (A) glyphosate, (B) paraquat e (C) amônio-glufosinato.

\section{Conclusões}

Com base nos resultados do presente estudo pode-se concluir que a aplicação do glyphosate em jato dirigido não prejudicou o desenvolvimento inicial das plantas, independentemente da dose utilizada. Entretanto, o contato do paraquat e do amônioglufosinato com o caule e folhas do baixeiro prejudica o desenvolvimento inicial das plantas de pinhão-manso.

\section{Referências}

ACHTEN, W.M.J. et al. Jatropha bio-diesel production and use. Biomas and Bioenergy, v.32, n.12, p.1063-1084, 2008.

BECKIE, H.J.; REBOUD, X. Selecting for weed resistance: herbicide rotation and mixture. Weed Technology, v.23, n.3, p.363-370, 2009.

COSTA, N.V. et al. Directed-spray application of paraquat and diuron in physic nut plants. Planta Daninha, v.31, n.4, p.987-996, 2013. 
COSTA, N.V. et al. Efeito da deriva simulada de glyphosate no crescimento inicial de plantas de pinhão-manso. Planta Daninha, v.27, n.esp., p.105-110, 2009.

CHRISTOFFOLETI, P.J.; LÓPEZ-OVEJERO, R. Principais aspectos da resistência de plantas daninhas ao herbicida glyphosate. Planta Daninha, v.21, n.3, p.507-515, 2003.

DIVAKARA, B.N. et al. Biology and genetic improvement of Jatropha curcas L.: A review. Applied Energy, v.87, n.3, p.732-742, 2010.

DUARTE JÚNIOR, J.B. et al. Períodos de convivência das plantas daninhas com a cultura do pinhão manso (Jatropha curcas L.). XXI Congreso Latinoamericano de Malezas y XXXIV Congreso Mexicano de la ASOMECIMA, 2013. Anais... Cancún, México, 2013, p. 671-675. (CD-ROM).

ERASMO, E.A.L. et al. Tolerância inicial de plantas de pinhão-manso a herbicidas aplicados em pré e pós-emergência. Planta Daninha, v.27, n.3, p.571-580, 2009.

FEY, R. et al. Identificação e interferência de plantas daninhas em pinhão-manso. Revista Brasileira de Engenharia Agrícola e Ambiental, v.17, n.9, p.955-961, 2013.

GONÇALVES, K.S. et al. Seletividade de herbicidas aplicados em pós-emergência em pinhão manso (Jatropha curcas L.). Revista Brasileira de Herbicidas, v.10, n.2, p.110-120, 2011.

KUMAR, A.; SHARMA, S. An evaluation of multipurpose oil seed crop for industrial uses (Jatropha curcas L.): A review. Industrial Crops and Products, v.28, n.1, p.1-10, 2008.

MACIEL, C.D.G. et al. Possibilidade de aplicação de misturas de herbicidas de ação total com jato dirigido em mamoneira de porte anão. Planta Daninha, v.26, n.2, p.457-464, 2008.

OPENSHAW, K. A review of Jatropha curcas: an oil plant of unfulfilled promise. Biomass \& Bioenergy, v.19, n.1, p.1-15, 2000.
ROCHA, P.R.R; et al. Seletividade de herbicidas pré-emergentes ao pinhão-manso (Jatropha curcas). Planta Daninha, v.28, n.4, p.801-806, 2010.

SATURNINO, H.M. et al. Cultura do pinhão manso (Jatropha curcas L.). Informe Agropecuário, v.26, n.229, p.44-78, 2005.

SEVERINO, L.S.; VALE, L.S.; BELTRÃO, N.E. A simple method for measurement of Jatrorpha curcas leaf area. Revista Brasileira de Oleaginosas e Fibrosas, v.11, n.1, p.9-14, 2007.

SILVA, J.I.C. et al. Períodos críticos de interferência de plantas daninhas na cultura do pinhão manso submetido à poda. XXI Congreso Latinoamericano de Malezas y XXXIV Congreso Mexicano de la ASOMECIMA, 2013. Anais... Cancún, México, 2013, p.838-843. (CD-ROM).

SOCIEDADE BRASILEIRA DA CIÊNCIA DAS PLANTAS DANINHAS - SBCPD. Procedimentos para instalação, avaliação e análise de experimentos com herbicidas. Londrina: $1995.42 \mathrm{p}$.

VENCILL, W.K. et al. Herbicide resistance: toward an understanding of resistance development and the impact of herbicideresistant crops. Weed Science, v.60, n.esp., p.230, 2012. 